PHILIPPE BOURDIN

ORCID: 0000-0001-9950-9242

Université Clermont Auvergne

Institut Universitaire de France

philippe.bourdin@uca.fr

\title{
THÉÂTRE ET SOCIÉTÉS DANS LA FRANCE DE LA RÉVOLUTION
}

La véritable théâtromanie des Lumières est marquée tant par l'élévation de nouvelles salles au cœur de villes embellies par Soufflot, de Wailly, Louis, Lenoir-le-Romain ou Crucy $^{1}$, que par la diversification des principaux acteurs du monde des spectacles. Les entrepreneurs privés et les sociétés d'actionnaires accompagnent ou précèdent l'investissement des pouvoirs publics locaux et de l'État, qui consacrent de plus en plus cette libre entreprise. Les deux privilèges publics des spectacles, royal et municipal, s'ils coexistent (non sans accroc), sont donc concurrencés par les " privilèges " privés détenus par des particuliers et/ ou des actionnaires réunis en société, qui investissent l'espace culturel et marchand des spectacles ${ }^{2}$. Cette mutation structurelle tend à renouveler les publics, posant de facto de manière accrue la question de la police des spectacles. La sociabilité des salles publiques ne peut cependant à elle seule résumer les us, les coutumes et les réseaux entretenus par la curiosité et la pratique théâtrale : les sociétés d'amateurs se multiplient elles aussi, cercles de société révélateurs d'un entre-soi ouvrier, paysan, bourgeois ou aristocratique. La Révolution française bouleverse

${ }^{1}$ D. Rabreau, «Le théâtre-temple ou la trilogie des espaces », [dans :] De l'esprit des villes. Nancy et l'Europe urbaine au siècle des Lumières, A. Gady, J.-M. Pérouse (dir.), Éditions Artlys, Versailles 2005, p. 191 ; D. Rabreau, Le Théâtre et l'embellissement des villes en France au XVIII siècle, [thèse de doctorat], Université Paris-IV-Sorbonne, Paris 1978.

2 L. Clay, « Patronage, Profits, and Public Theaters: Rethinking Cultural Unification in Ancien Régime France », The Journal of Modern History 4, vol. 79, 2007, pp. 729-771. 
ces différents états, en décrétant la liberté commerciale pour les entrepreneurs de spectacles par la loi Le Chapelier du 13 janvier 1791, en faisant plus que jamais des salles des chambres d'écho des débats et des luttes publics, en associant les sociétés dramatiques au militantisme des clubs jacobins ou de la réaction thermidorienne - ce qui impose à leurs membres et une plus grande mixité sociale et la publicité de leurs activités. Derrière la question du théâtre et des sociétés en Révolution, il y a donc celle des sociétés d'actionnaires, celle des amateurs, celle enfin des spectateurs.

\section{LES SOCIÉTÉS D’ACTIONNAIRES}

La liberté commerciale privée donnée aux entrepreneurs de spectacles par la loi du 13 janvier 1791 consacre une transformation engagée depuis les années 1770 en province. Les autorisations exceptionnelles accordées dans la capitale l'avaient déjà amorcée dès la décennie 1760 : pensons aux nouveaux espaces ouverts sur les boulevards, tels les théâtres de Nicolet, d'Audinot, des Associés, des Élèves de l'Opéra et des Délassements Comiques. Le phénomène s'était accéléré dans les années 1780 : les Théâtres de Beaujolais à partir de 1783, des Variétés Amusantes de 1787 puis de Monsieur depuis 1789, fonctionnaient comme des entreprises privées. Les abolitions des privilèges et des monopoles pour les représentations parisiennes des 4 août 1789 et 26 août $1790^{3}$ avaient anticipé la loi du 13 janvier 1791, qui abolit de fait le privilège des grandes scènes parisiennes (Opéra, OpéraComique, Comédie-Française). Elle autorise « tout citoyen à élever un théâtre public et y faire représenter des pièces de tout genre » en en faisant la simple

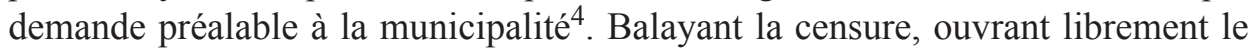
répertoire aux œuvres des dramaturges morts depuis dix ans et sous conditions à celles des auteurs contemporains, cette législation libératrice consacre le théâtre comme entreprise commerciale privée ${ }^{5}$.

Les salles et les troupes se multiplient alors, et les pratiques dramatiques gagnent des espaces jusque-là peu familiarisés au divertissement. Dans la capitale, l'effet est immédiat : les trois théâtres Molière, Louvois et du Marais accueillent leurs premiers spectateurs entre le 18 juin et le $1^{\text {er }}$ septembre 1791 ; puis les ouvertures se multiplient au point que Paris compte près de trente-cinq lieux

${ }^{3}$ M. de Rougemont, La vie théâtrale en France au XVIII siècle, Slatkine Reprints, Genève 1988, p. 286.

${ }^{4}$ Loi du 13 janvier 1791. Article 1 : « Tout citoyen pourra élever un théâtre public, y faire représenter des pièces de tous les genres, en faisant préalablement à l'établissement de son théâtre, sa déclaration à la municipalité des lieux ».

5 P. Frantz, «Le théâtre sous l'Empire : entre deux révolutions », [dans :] L'Empire des muses. Napoléon, les arts et les lettres, J.-C. Bonnet (dir.), Belin, Paris 2004, p. 174 ; L. Clay, op. cit., pp. 729-771. 
publics de spectacle en 1792, et une vingtaine sont toujours actifs en 1797. En province, neuf théâtres au moins voient le jour entre 1750 et 1759 , seize entre 1760 et 1769, dix-neuf entre 1770 et 1779 , vingt-quatre entre 1780 et 1790, vingt-neuf au moins dans les quinze années suivant la loi Le Chapelier — dont ceux d'Angers (1791), de Béziers (1792), de Carcassonne (1796), de Nîmes (1801). La pédagogie théâtrale républicaine promue par les cercles jacobins en l'an II explique toutefois directement la nette hausse des constructions entre 1793 et 1795, la plus importante depuis le milieu du siècle à Paris et en province.

Dans un désir d'oubli de ces investissements patriotiques de l'an II et des luttes violentes qui se multiplient au sein ou à l'extérieur des salles de spectacle, la liberté théâtrale est à nouveau en débat sous le Directoire. La motion d'ordre sur les théâtres, présentée le 26 brumaire an VI (16 novembre 1797) par Marie-Joseph Chénier au Conseil des Cinq-Cents ${ }^{6}$, abonde dans ce sens. Surveillance et régulation de la libre activité des théâtres sont à l'ordre du jour, le père du Charles IX voyant même en la multiplicité des scènes une dangereuse source d'anéantissement de l'art dramatique. Bien que le projet de loi du 18 prairial an VI (6 juin 1798) soit finalement ajourné, l'idée d'une réduction du nombre de théâtres, à Paris et en province, est avancée. Mais, c'est bien quelques années après qu'elle prend corps. Les premières tentatives ont lieu sous le Consulat : réorganisation des scènes lyriques à Paris, fermeture de petites salles bâtardes en province, à Rouen ou à Lyon. Napoléon réhabilite ensuite le système de l'ancien privilège monarchique. Il contrôle et réduit le nombre de salles autorisées dans les grandes villes par l'adoption du Règlement impérial pour les théâtres du 8 juin 1806, ce qui n'empêche pas l'ouverture de cinquante nouveaux lieux provinciaux entre 1808 et 1817.

À partir des années 1760-1770, la nouvelle forme juridique, économique et sociale, la plus significative de l'engagement privé dans la construction et la gestion des lieux de spectacles, est bien celle de la société par actions. À Rochefort (1767), Grenoble (1768), Le Mans (1775), Rouen (1776), Reims (1777), Lorient (1778), Lille (1784), Saumur (1785), Marseille (1787) ${ }^{7}$, le modèle fait flores et se décline ailleurs encore pendant la Révolution puis sous l'Empire. Il n'est pas sans risques. Ainsi en va-t-il à Nantes. L'instabilité chronique des directions des trois salles nantaises, à commencer par le flambant neuf Grand Théâtre de Mathurin Crucy, inquiète les acteurs et leurs financeurs. Les premiers, réunis en société, échouent année après année à stabiliser leur activité. La désignation d'un nouvel homme fort, Danglas, à partir de 1793, procède surtout du lobbying exercé par les grands négociants nantais. Rapidement pourtant, le siège de Nantes et les événements vendéens aboutissent à des fermetures temporaires qui ont raison

${ }^{6}$ M.-J. Chénier, Motion sur les Théâtres, Conseil des Cinq-Cents, séance du 26 brumaire an VI, p. 2.

7 L. Clay, op. cit. 
et des finances du principal théâtre, et de ses équipes de direction successives. L'incendie du bâtiment, le 7 fructidor an IV (25 août 1796), prive les Nantais du lieu de divertissement le plus moderne de la ville. Il les oblige à se rabattre sur les deux anciennes salles du Bignon-Lestard et du Chapeau Rouge. Là est l'occasion pour les acteurs de la vie théâtrale locale d'imaginer un nouveau mode d'association pour envisager la reconstruction du Grand Théâtre et sa meilleure gestion (ce, dès le 31 août 1796). Ils rédigent un Projet d'établissement proposé pour la reconstruction de la salle de spectacle de Nantes ${ }^{8}$, permis par une société au capital espéré de 1,2 millions de livres et selon un modèle de développement économique structurel déjà éprouvé ailleurs.

Souvent, une centaine d'investisseurs au moins se retrouve en versant une contribution minimum ; à Reims, 116 particuliers détiennent ainsi les 156 actions du capital de la société. La formule la plus usitée est celle de la tontine, qui offre un revenu permanent pour un investissement principal initial ; le retour sur investissement est alors conditionné par les ressources dégagées par l'exploitation de la salle et les actions se transmettent aux descendants de leurs propriétaires s'ils n'ont pas décidé de les remettre en vente avant la fin de leur existence. Aussi certains actionnaires héritiers bénéficient-ils de l'investissement initial de leurs parents, comme c'est le cas à Lille par-delà la Révolution et l'Empire. Ces sociétés ne se contentent toutefois pas seulement de lever des fonds et de veiller à la concrétisation de chantiers majeurs, elles participent aussi à la gestion directe de ces salles pendant plusieurs décennies. Elles se caractérisent par une ouverture sociale certaine. En 1789, au Mans, les 108 actionnaires se répartissent entre 36 nobles, 31 hommes de loi et officiers royaux et municipaux, 32 négociants et marchands, mais aussi quelques artisans d'art et/ou artistes (un architecte, un horloger, un peintre notamment). À Lille, cent actions sont mises en ventes et achetées par quelque 76 individus, parmi lesquels 32 nobles et 4 veuves héritières d'aristocrates, 8 hommes de loi et officiers publics, 20 négociants, mais aussi 1 maître couvreur, 1 plombier et 1 chirurgien, 2 brasseurs, 1 imprimeur, 1 libraire et 1 maître de musique. Malgré la diversité des actionnaires, le privilège de la fortune et du rang impose au groupe une plus forte représentation des nobles, détenteurs souvent de plusieurs actions ${ }^{9}$.

${ }^{8}$ Archives municipales de Nantes, 2R583, Projet d'établissement proposé pour la reconstruction de la salle de spectacle de Nantes et l'achèvement de la Bourse avec ses accessoires, le 14 fructidor an V (31 août 1796).

9 H. Hennebel, Le théâtre à Lille dans le second XVIIIe siècle, [maîtrise de l'Université Lille III], Lille 1999, pp. 170-175. Les grandes figures de l'administration locale sont représentées, avec les Imbert, alors depuis quarante-cinq ans aux affaires publiques locales, quelques visages connus de l'élite intellectuelle et savante lilloise avec les Danel, Panckoucke et Jacquez. Plusieurs grandes familles nobles, par ailleurs habituées du spectacle, sont, elles aussi, représentées par les personnes des comtes de Muisseret et de Sainte Aldegonde, du vicomte de Mailly, des marquis d'Aigremont et de la Riandrie, ou des seigneurs de Zurgault, de Savreuil ou d'Anstaing. 
La Révolution change la donne. Les sociétés d'actionnaires reculent devant l'incertitude des événements : ainsi à Bordeaux en 1793, où plusieurs négociants renoncent à leurs investissements culturels. La sociologie des sociétés d'actionnaires reflète les changements socio-politiques : la nouvelle société nantaise par actions montée dans l'été 1796 compte des représentants de la bourgeoisie négociante, des avocats, des médecins, des officiers publics et des hommes à talents qui marquent ainsi leur leadership civique, économique et culturel. Cela ne signifie nullement un effacement complet de la noblesse : à Aurillac, en l'an VII, on retrouve parmi les 89 actionnaires qui financent l'aménagement d'une nouvelle salle de spectacles 6 ex-nobles, récents acquéreurs de grands domaines, 31 représentants des professions libérales — de santé et de justice - 19 négociants, marchands et commerçants, 16 administrateurs et fonctionnaires civils, 8 propriétaires, 6 artisans, 4 ingénieurs, techniciens ou experts, 3 militaires et autant d'ecclésiastiques ${ }^{10}$. De fonctionnement démocratique et le plus souvent égalitaire, votant sur les décisions les plus importantes ou pour désigner des commissaires annuellement renouvelés, ces réunions d'intérêts s'intéressent évidemment au sérieux de la gestion financière, qui dépend de la permanence et de la programmation des troupes. D'où leur influence sur le choix du directeur du spectacle, des premiers artistes et du répertoire : ainsi, en 1790, les actionnaires du Grand Théâtre de Marseille refusent de céder à la demande des spectateurs de recruter à nouveau les époux Ponteuil, partis à l'intersaison précédente ${ }^{11}$. Ils mettent également tout en œuvre pour obtenir la libre représentation de l'opéra-comique Les Visitandines, finalement accordée par les auteur et compositeur Picard et Devienne en octobre $1792^{12}$. Il faut cependant à ces sociétés trouver un modus vivendi avec les municipalités qui partagent les mêmes prétentions - et peuvent progressivement racheter les actions (à Reims, où la salle a été ouverte en 1777, la municipalité ne devient majoritaire parmi les investisseurs qu'en $1792^{13}$ ).

Le théâtre, qui peut s'avérer rémunérateur, devient le creuset de véritables reconversions sociales. Il en va ainsi pour Élisabeth Blondeau de Garron, dont le père et les deux frères ont été tués dans les guerres de Vendée, ce qui la conduit en 1800, à l'âge de 25 ans et grâce à une petite expérience théâtrale engrangée les années précédentes, à devenir directrice de spectacle : si elle a été « élevée à l'école du malheur », selon ses propres termes, les Cent-Jours signeront la ruine de son entreprise ${ }^{14}$. Le chevalier Cantiran de Boirie a lui aussi fréquenté les mêmes

10 C. Triolaire, La vie théâtrale en province, Presses universitaires Blaise Pascal, ClermontFerrand 2012, pp. 420-421.

11 Archives municipales de Marseille, 1I550, Lettre des actionnaires au couple Ponteuil, le 18 octobre 1790 .

12 Archives municipales de Marseille, 1I560, Accord de libre représentation, le 20 octobre 1792.

13 Archives municipales de Reims, 5N17, Pétition, le 2 floréal an IV (21 avril 1796).

14 Archives nationales /F 17/1299/E, Lettres au roi, au comte d'Artois, à la duchesse d'Angoulême, et au ministre de l'Intérieur, s. d. [1815]. 
bancs, arrêté par le Vol de l'Aigle alors qu'il venait d'acquérir la salle parisienne des Jeunes Artistes, fruit d'une carrière théâtrale commencée sous la Révolution pour sauver une famille privée de quarante mille livres de rentes, dont dix-sept membres sont morts pour la cause des princes (et parmi eux treize chevaliers de Saint-Louis) ${ }^{15}$. François-Robert Vée-Duchaume, qui brille à Dijon et à Besançon en faisant venir les «premiers artistes de la capitale», tout en gérant plus difficilement les salles de Gray et de Vesoul, est l'un des anciens marchands de vin du roi, d'abord fondé de pouvoirs du directeur Borsary avant d'en reprendre le privilège ; lui et sa femme, l'un comme basse-taille, l'autre comme première Dugazon et St. Aubin, jouent dans la troupe pour faire des économies de salaires ${ }^{16}$. Sans parler des familles de planteurs ruinées par les révoltes et les guerres. Émilie Baudin, issue d'une famille de Saint-Domingue ayant tout perdu dans le soulèvement des esclaves en 1791, se met ainsi en 1807 sous la protection de Charles Pougens, qui excipe de ses titres (Institut de France, académies étrangères) et de l'assurance que sa jeune protégée jouit $d^{\prime}$ ' une tête bien organisée ${ }^{17}$. Elle, qui a fréquenté cinq ans durant seulement des sociétés d'artistes dramatiques, argue surtout de sa " créolité », qui la rapproche de Joséphine de Beauharnais ${ }^{18}$.

Mais la Révolution est aussi porteuse de risques politiques. Les engagements politiques divisent les troupes, font peser sur les épaules des uns ou des autres le poids de la suspicion (ainsi des directeurs du Vaudeville, dénoncés en 1793 par la Feuille de salut public), et interrompent des carrières. Tel est le cas de Léonard Autié, protégé de Marie-Antoinette, ancien directeur du Théâtre de Monsieur, d'abord relégué à la foire Saint-Germain en 1791, puis brutalement contraint de partir en juin 1792. Signant sous la contrainte la cession de ses propriétés, il conduit sur les routes européennes, à ses frais et à ses risques, une partie du trésor royal (une cassette de diamants) ; il sollicitera en vain le rétablissement de ses prérogatives sous la Restauration ${ }^{19}$. Les risques financiers ne sont pas moins grands : la guerre enlève à la scène des artistes atteints par les exigences de la conscription, elle détruit aussi des salles, ou conduit à leur pillage sur la frontière est du pays ; quant à l'occupation de territoires étrangers, elle ne permet jamais aux entrepreneurs qui croient y trouver des débouchés de s'installer durablement, les incertitudes de la victoire, l'obstacle de la langue et le manque de matériel jouant contre eux. Partout, et d'abord en France, il faut enfin compter avec la concurrence des

15 Archives nationales /F 17/1299/E, Lettre de Boirie au ministre de l'Intérieur, depuis Paris (24, rue Notre-Dame de Nazareth), 26 juin 1816.

16 Archives nationales /F 17/1299/E, Lettre de Vée Duchaume au ministre de l'Intérieur, s. d. [août 1814].

17 Archives nationales /F 17/1299/D, Lettre de Charles Pougens au ministre de l'Intérieur, 5 octobre 1807.

18 Archives nationales /F 17/1299/D, Lettre d'Émilie Baudin au ministre de l'Intérieur, s. d. [1807, puisqu'elle mentionne et associe la recommandation précédente de Pougens].

19 Archives nationales /F 17/1299/D, Lettres adressées par Autié au ministre de l'Intérieur (8 décembre $1817 ; 6$ juillet 1818). 
sociétés d'amateurs, qui ont le soutien des autorités publiques, bénéficient de priorités dans l'occupation des salles, et imposent leurs contraintes aux professionnels - certains de ces derniers préfèrent un temps accepter l'amalgame (à Dijon) ou sont contraints de partager leur temps avec un second métier (à Nantes).

\section{LES SOCIÉTÉS D’AMATEURS}

Malgré l'incertitude de leur sort politique, l'exemple artistique des professionnels et des grandes scènes subventionnées parisiennes stimule en effet un monde d'amateurs, réunis depuis la deuxième moitié du siècle en sociétés dramatiques. Comme la fréquentation assidue des salles, ces associations sont l'une des traductions de la théâtromanie qui s'est emparée des Français depuis au moins le mitan du siècle. Les objets dérivés de la scène participent de cet engouement : n'aidentils pas à reconnaître les moments cruciaux de la dramaturgie, les répliques les plus signifiantes, peintes sur des éventails que l'on s'arrache rue Saint-Jacques ${ }^{20}$, voire sur les broderies des gilets et les boutons des vestes masculines? Ils contribuent à construire le vedettariat d'artistes majeurs dont circulent des gravures (une troupe entière pouvant offrir les figures d'un jeu de cartes), des biographies fantaisistes, jusqu'aux critiques et compliments que leur adressent les particuliers par journaux ou almanachs interposés ${ }^{21}$. Qu'il ait bénéficié de la pratique théâtrale initiée par les jésuites, du théâtre de société des cercles bourgeois ou populaires (jusqu'aux concours annuels des communautés paysannes de Flandre ${ }^{22}$ ), ou encore du théâtre d'éducation dans lequel s'illustre $\mathrm{M}^{\mathrm{me}}$ de Genlis ${ }^{23}$, l'amateur éclairé des arts de la

${ }^{20}$ G. Letourmy, «L'éventail du succès : le théâtre mis en images au début de la Révolution », [dans :] La scène bâtarde entre Lumières et Romantisme, Ph. Bourdin, G. Loubinoux (dir.), Presses Universitaires Blaise Pascal, Clermont-Ferrand 2004, pp. 221-240.

21 On peut ainsi lire encore dans le Journal de Paris du 17 pluviôse an III (5 février 1795) ce compliment à $\mathrm{M}^{\text {lle }}$ Contat : « La fable apprend que l'empire comique, / Autrefois à Thalie en lot étoit échu ; / En ce moment la vérité s'explique, / Et prouve qu'à Contat cet apanage est dû ».

22 G. Hérelle, Les Théâtres ruraux en France, Champion, Paris 1930. Marquant la valeur religieuse et communautaire, son rôle de conservatoire des idiômes et des cultures (dans les pays de langue d'oc, en Savoie, dans le Dauphiné, en Bretagne ou en Flandre), l'auteur en fait un héritage du Moyen Âge. Ce théâtre se fond parfois dans les carnavals, les charivaris, les mystères, ou donne lieu en Flandres à des concours laïcs organisés autour d'une seule pièce classique réinterprétée par plusieurs troupes, en langue vernaculaire.

${ }^{23}$ Cf. M.-E. Plagnol-Dieval, Madame de Genlis et le théâtre d'éducation au XVIII siècle, Oxford 1997. Ce théâtre d'éducation est l'objet de conséquentes publications des années 1750 à la Révolution : travaux de $\mathrm{M}^{\text {mes }}$ de Maintenon, de La Fite, de Genlis, du marquis de Saint-Marc, de Berquin, de Nougaret, de Gessner, de Garnier ou de Moissy. Ces œuvres, réécritures rapides de thèmes connus, ne donnent lieu à aucune représentation publique sur une scène officielle, privilégiant l'intimité du pensionnat, du cercle familial, du salon — sauf chez $\mathrm{M}^{\mathrm{me}}$ de Genlis, jouant plusieurs mois, accompagnée de ses deux filles, dans le théâtre loué d'une société bourgeoise où se pressent jusqu'à cinq cents spectateurs, parfois payants : un narcissisme dont $\mathrm{M}^{\mathrm{me}}$ de Staël appelait à se méfier dans La Sunamite. Démonstratif et explicatif, un tel théâtre contribue à faire glisser la 
scène fait son miel dans la France des Lumières puis dans celle de la Révolution d'abondantes publications : critiques de la presse provinciale et parisienne, ouvrages spécialisés (Almanach des Muses, Almanach des Spectacles, Courrier des spectacles, Journal des théâtres, Censeur dramatique, etc.). Demeurent des mémoires édulcorés mais instructifs sur les clés d'un apprentissage, d'un métier, des emplois, du répertoire et les limites des modèles et des solidarités. Ainsi des Élémens de l'art du comédien, de Dorfeuille, dit Poupart (an VII-an VIII), qui marie classicisme - dans sa revendication d'une école de déclamation, déjà tentée en 1784 - et modernité, par sa conception voltairienne d'une appropriation et d'une intériorisation du texte et de son rôle par l'acteur, nourricier de l'illusion, suppléant à l'incomplétude de la langue ${ }^{24}$. C'est ce statut de créateur que revendiquent Talma ou $\mathrm{M}^{\text {lle }}$ Clairon, acteurs de la libération du costume et du geste $^{25}$. La deuxième publie en 1798 les Mémoires d'Hyppolyte Clairon, et réflexions sur l'art dramatique. Parmi ses élèves, l'avocat et Conventionnel Hérault de Séchelles, qui a livré lui-même en 1794 ses Réflexions sur la déclamation ${ }^{26}$ considérations sur la mémorisation, la concentration, l'attitude, le regard, le ton et le geste de l'orateur.

Il n'est pourtant pas unanimement admis dans la France de 1789 que l'on puisse se passionner pour le théâtre, fustigé par Rousseau dans sa Lettre à d'Alembert sur les spectacles (1758). Contre les amoureux de la scène tonnent encore quelques suppôts de l'Église, prompts à excommunier les artistes et leurs thuriféraires livrés à cet enfer des passions. L'abbé Parisis, sur la foi de nombreux mandements épiscopaux (des prélats d'Amiens, d'Arras, de Cambrai, de Mâcon et de Toulon notamment), ou à la lecture du Discours sur les Spectacles du Père Charles Porée (1732), confond la comédie, notamment celle des Italiens, avec « la fange des vices » agitée par Satan, meurtre et plaisir mêlés. Il faut détourner les spectateurs de cette « école de l'impureté » en leur refusant l'absolution et les acteurs en maintenant leur excommunication, tous coupables de péché mortel ${ }^{27}$. En juillet 1789, le Journal ecclésiastique (pp. 279-280) vitupère contre « la foule de petits théâtres si propres à augmenter la licence, le libertinage des mœurs comme des opinions, et à en devenir une école publique pour tous les âges, ainsi que pour toutes les conditions », que l'on a laissé se développer à Paris comme en province.

morale édifiante de la sphère religieuse à l'espace laïc. À travers intrigues et personnages-types, il oppose avec manichéisme et naïveté le bon et le méchant, la vertu et le vice, dans une suite éducative — les pièces renvoyant les unes aux autres — qui tient compte de l'âge des destinataires, des acteurs amateurs et valorise la civilité familiale.

${ }^{24}$ S. Chaouche (dir.), Écrits sur l'art théâtral (1753-1801), vol. II : Acteurs, Honoré Champion, Paris 2005, p. 566.

25 S. Marchand, Théâtre et pathétique au XVIII' siècle : pour une esthétique de l'effet dramatique, Honoré Champion, Paris 2009, pp. 550-551.

26 Ibidem, pp. 488-506.

27 Abbé Parisis, Questions importantes sur la comédie de nos jours, Valenciennes 1789 (deuxième édition). 
En l'an III encore, les Annales de la religion n'auront pas de mots assez durs contre les expériences de l'an II, élevant le théâtre au rang d'école des mœurs, et les comédiens, acteurs ou auteurs, à celui de prêtres de la morale publique ${ }^{28}$. De fait, les préventions contre les arts de la scène en entretiennent depuis longtemps d'autres, plus violentes, contre comédiens et comédiennes, dont la vie est supposée dissolue.

La distinction sociale conférée par la connaissance des jeux et répertoires de la scène est pourtant indubitable. Une partie de la bourgeoisie française y prétend obstinément. À elle les éventuelles pratiques privées d'un théâtre démonstratif et explicatif, réécriture de thèmes connus mobilisant intrigues et personnages manichéens pour mieux repérer le bon et le méchant, la vertu et le vice, selon les canons de la morale édifiante. À elle surtout le théâtre des collèges, celui des jésuites pour les pères de famille désormais aux responsabilités, celui des institutions en partie seulement renouvelées pour les plus jeunes, qui ouvrent enfin leur art au public le plus large et non plus aux seuls parents d'élèves. À Aurillac, des jeunes gens, du reste encouragés par un directeur de troupe, profitent en octobre 1789 de la désaffection du Présidial par les magistrats pour en bouleverser l'architecture et édifier une grande salle de spectacles, au mépris du danger d'incendie ${ }^{29}$. L'effondrement du cuvage servant de salle de représentation, le 20 décembre 1791 à ClermontFerrand, nous révèle là encore l'existence d'une troupe d'écoliers, victimes en la circonstance de leur succès ${ }^{30}$. Au Mans, de jeunes citoyens donnent, en janvier 1790, La Mort de César au théâtre municipal ; en l'an II, alors que les jacobins contrôlent les amateurs du cru, les collégiens sont encore assidûment présents ${ }^{31}$. Le théâtre est, du reste, étroitement intégré aux exercices scolaires promus par la Société des Jeunes Français de Léonard Bourdon, associant morale civique, arts mécaniques et divertissements comme la quintessence de la vie citoyenne : un spectacle dont ils sont les héros initie donc les élèves au fonctionnement d'une petite république, d'un tribunal citoyen, d'une fête républicaine, puis d'un atelier artisanal (celui du boulanger, de l'imprimeur, du menuisier, du cordonnier, etc.), qu'ils quittent pour se faire violonistes ou choristes ${ }^{32}$. Sous le Directoire encore,

28 Annales de la religion, 20 floréal an III (9 mai 1795), p. 29.

29 Archives communales d'Aurillac, I D (1), Délibération du 28 octobre 1789. Cf. C. Grimmer, Histoires de théâtre à Aurillac, Musée d'art et d'archéologie d'Aurillac, Aurillac 2001, p. 10.

${ }^{30}$ Bibliothèque Clermont Université, 180620 : Le Thermomètre du jour, journal de Dulaure, $\mathrm{n}^{\mathrm{os}}$ du lundi 26 décembre 1791 et du mercredi 18 janvier 1792 ; Bibliothèque intercommunautaire de Clermont-Ferrand, Patrimoine, Ms 928 : Lettre de l'élève Massonnet à son père, 18 décembre 1791. $C f$. Ph. Bourdin, Des lieux, des mots, les révolutionnaires. Le Puy-de-Dôme entre 1789 et 1799, Presses universitaires Blaise Pascal, Clermont-Ferrand 1995, pp. 52-53.

${ }^{31}$ K. Large, Les théâtres de Nantes et du Mans pendant la Révolution française (1789-1799), [mémoire de maîtrise], Université Blaise-Pascal 2001, p. 130.

32 Archives nationales, $\mathrm{F}^{17} 1331^{\mathrm{B}}$, Rapport des députés de la Commission des Arts [Lindet et Plaichard] pour assister aux exercices des Écoles républicaines de la Société des Jeunes Français, 9 messidor an II (27 juin 1794). 
les autorités, qui organisent une réaction républicaine au danger royaliste exprimé dans les urnes en l'an V, ne voient pas d'un mauvais œil la mobilisation des jeunes citoyens. Ainsi à Courrières, où des adolescents souhaitent s'adonner à la comédie : « rien ne s'oppose à leur désir, pourvu néanmoins que leurs pièces soient patriotiques, rendues les décadis et fêtes nationales, et qu'au besoin elles vous soient communiquées à l'avance », consent le commissaire auprès de l'administration départementale du Nord ${ }^{33}$. À Périgueux, pour la fête de la Vieillesse, il est entendu qu'aux frais de la municipalité « de jeunes élèves joueront une comédie et un opéra $»^{34}$.

La Révolution favorise la création des sociétés dramatiques, quittant la sphère privée, pour un public élargi et divers : anciens cercles bourgeois mis au service des opinions de leurs animateurs, promus au sein des municipalités et des clubs (Riom); créations ex-nihilo par les Jacobins, par les sociétés populaires (Besançon, Dijon) ${ }^{35}$, par les administrations locales sous la réaction thermidorienne et le Directoire (Albi, Troyes, Tulle $)^{36}$; divertissements utiles reproduits au sein des armées françaises enfin ${ }^{37}$. En décembre 1793, les Jacobins de la capitale demandent au comité d'Instruction publique de faire décréter à la Convention « que dans chaque ville de 4000 âmes il y ait une salle de spectacles où les élèves des écoles publiques et d'autres

33 Archives départementales du Nord, L 489 (1), Lettre du commissaire du département du Nord à son collègue de Courrières, 19 ventôse an VII ( 9 mars 1799).

34 Archives départamentales de la Dordogne, E DEP 5209, Délibérations municipales, séance du 6 fructidor an V (23 août 1797).

35 Michael L. Kennedy en fait brièvement mention dans sa synthèse sur les clubs jacobins français : il cite les sociétés dramatiques de Douai, Chaumont, Poligny, Vesoul, Lectoure, Pertuis, Limoges, Chartres (purgée des individus « immoraux et aristocrates ») - M.L. Kennedy, The Jacobin Clubs in the French Revolution (1793-1795), Berghahn Books, New York 2000, pp. 194-195.

${ }^{36} \mathrm{Ph}$. Bourdin, "Le théâtre, les amateurs, la Révolution », [dans :] La scène bâtarde entre Lumières et Romantisme, Ph. Bourdin, G. Loubinoux (dir.), Presses Universitaires Blaise Pascal, Clermont-Ferrand 2004, pp. 241-254 ; idem, "Les théâtres de société sont-ils solubles dans la Révolution ? L'exemple de l'Auvergne », Études sur le XVIII siècle 33 (« Les théâtres de société au XVIII siècle »), Éditions de l’Université libre de Bruxelles, Bruxelles 2005, pp. 157-168; idem, " Drama societies in the French Revolution: from jacobin enthusiats to royalist amateurs », French History 1, vol. 22, Oxford 2008, pp. 3-27 ; A. Hertert, « La Société dramatique de Besançon et le personnel révolutionnaire bisontin de 1793 à 1796 », [dans :] Théâtre et Révolution, numéro spécial des Annales littéraires de l'Université de Besançon, Paris 1989, pp. 35-43 ; C. Tréhorel, Le théâtre à Dijon de 1789 à 1810, [mémoire de maîtrise], Université de Dijon, 1999 ; Archives départementales de Corrèze pour Tulle (1 E DEP 271/831. Registres de la société dramatique) ; Archives départementales de Vendée pour Fontenay-le-Comte (L 1364. Registre de délibérations de la société populaire du cru), Archives départementales du Tarn pour Albi (L 740, f $^{\circ}$ ).

37 Capitaine Duthilt, Mes campagnes et mes souvenirs, Le Livre chez vous, Paris 2008, pp. 190-191. En 1801, à Bologne, les militaires français fondent un théâtre de société, sur lequel l'auteur versifie, non sans plaider l'indulgence des futurs spectateurs (« La lyre et le mousquet ont trop peu de rapport/ Pour que la même main les accorde d'abord ») : "Thalie et Melpomène excitent nos désirs / Et leurs jeux variés rempliront nos loisirs. / Par elles dirigés, sous les traits de la fable, / Nous pouvons réunir l'utile et l'agréable ». 
personnes puissent répéter et représenter seulement des œuvres édifiantes et dans l'esprit de la Révolution ${ }^{38}$. À moins qu'elles ne couvrent un vide territorial, à l'écart des tournées des troupes, ces sociétés évincent la plupart du temps des lieux où elles s'installent les professionnels, dont le répertoire est jugé insuffisamment républicain.

Elles ont souvent sur les troupes classiques l'avantage du nombre et de la jeunesse (au bénéfice des trentenaires), de la reconnaissance sociale et politique, de la solidité et du renforcement des liens maritaux, familiaux, amicaux, professionnels, maçonniques ou de voisinage. Administrateurs, hommes de loi, négociants, enseignants et praticiens, forts de leurs pratiques collégiennes des arts de la scène ou de leur savoir littéraire, dominent les sociétés. À Dijon, Nicolas Guillaume Bazire, frère du Conventionnel Claude Bazire, et Louis Viardot, ont été les meneurs de la révolution municipale en juillet 1789 , et treize citoyens fréquentent depuis son origine la société populaire. Les sociétés dramatiques souffrent en revanche d'une dichotomie sexuelle induite par leur horizon de recrutement (notamment dans les clubs masculins) et fort gênante pour la distribution des rôles. Elle est particulièrement forte à Tulle (12\% de femmes), à Riom (24\%), à Fontenay-leComte (31\%) et à Dijon ( $38 \%$ ). À Besançon, où $83 \%$ des artistes en moyenne sont des hommes, et parmi eux $11 \%$ d'adolescents, ce déséquilibre est accentué par la mésentente des militants avec la Société des Amies de la Liberté et de l'Égalité, fondée en 1792. Les saisies révolutionnaires chez les nobles et les prélats émigrés donnent l'opportunité de développer les magasins d'habits et les décors sans en pallier pour autant toutes les défaillances.

Fières de leurs missions propagandistes et philanthropiques (leurs recettes sont en général versées aux indigents), les sociétés d'amateurs copient les règlements associatifs des gens de l'art. Le règlement bisontin du 18 prairial an II (30 juin 1794) prévoit ainsi différentes conditions d'admission : des qualités morales appréciées par le comité de la section du candidat; des aptitudes au théâtre vérifiées par le comité d'examen de la société dramatique ; une élection par l'assemblée générale des amateurs et l'approbation des officiers municipaux. À Fontenay-le-Comte, en pleine Vendée insurgée, le règlement du 3 messidor an II (21 juin 1794) — le plus intransigeant pour l'heure découvert - relève du comité des fêtes décadaires, qui distribue les rôles, punit les amateurs ne les assumant pas, batifolant aux répétitions, ou y ajoutant « quelques expressions satyriques ou indécentes ». Il prévoit explicitement de censurer les pièces pour assurer l'œuvre de propagande. En coulisses comme dans la salle, il veille à la partition des sexes (réservant par exemple les loges aux seules citoyennes). Le texte riomois, du 7 pluviôse an III (26 janvier 1795), planifie les prérogatives de la direction, les rôles et les entrées des artistes, la gestion des répétitions et des défaillances (jusqu'aux amendes infligées pour

38 G. Radicchio, M. Sajous d'Oria, Les théâtres de Paris pendant la Révolution, L. Casati, F. Lenoir (trad.), Fasano 1990, p. 47. 
travail insuffisant ou absences, de 20 sols à 3 livres), l'emploi salarié du garçon de salle. Il affirme l'autogestion de l'ordre interne et la collégialité du choix du répertoire et du fonctionnement d'un ensemble au sein duquel l'individu est intégré par cooptation de cinq membres - cooptation qui renvoie aux modèles académiques et maçonniques. Les missions patriotiques, la participation aux fêtes civiques demeurent des exigences. La revendication du sérieux, le souci de protection morale des jeunes filles transparaissent d'un article à l'autre.

Toujours est-il que le modèle des grandes scènes parisiennes impose une disponibilité à toute épreuve à des citoyens et des citoyennes dont le théâtre n'est pas la seule raison de vivre, et qui se dévouent au sortir des heures de travail pour proposer leurs talents au plus grand nombre, paysans des environs compris - à partir de $16 \mathrm{~h}$ à Riom, à $17 \mathrm{~h}$ à Fontenay-le-Comte, à $19 \mathrm{~h}$ puis $18 \mathrm{~h} 30$ à Tulle. L'exemple de la société dramatique de Tulle, la seule pour laquelle nous possédions la distribution pour dix pièces, données en cinq représentations à partir de nivôse an III, est très révélateur des investissements individuels. Tous les membres ne montent pas sur scène (seuls 59\% tentent l'aventure, dont quatre couples), où se succèdent, après les deux pièces en trois actes du premier soir, à la durée évidemment trop longue, des comédies en trois actes et en un. Pour les quatre premières séries de représentations, eu égard aux aléas de la vie professionnelle, des doublures sont prévues. Tant et si bien que huit des vingt-neuf amateurs (les vedettes de la troupe, qui excellent dans les emplois de jeunes premiers ou de valets effrontés) peuvent apprendre de 8 à 10 rôles, neuf de 5 à 7 , six de 2 à 4 , six se contentant d'un !

Avec ces théâtres de société révolutionnaires, on reste néanmoins largement dans le domaine de la mondanité : ils s'interrompent une fois l'été venu, et les notables partis pour leurs résidences secondaires ; ils savent agglomérer des solidarités et susciter des modes - à Riom, " la Comédie bourgeoise fait fureur », peut ainsi noter Miette Tailhand, nièce du Conventionnel Gilbert Romme ${ }^{39}$. À l'heure des réseaux (de cercles érudits, de salons, de loges, de correspondances), il semble que des liens soient tissés d'une réunion d'amateurs à l'autre. Ceux de Tulle, qui ont besoin de décors de place publique et de maison rustique, font ainsi appel en floréal an III à un peintre qui a déjà travaillé pour la société dramatique de Limoges. Miette Tailhand assiste en 1793, à Issoire, ville commerçante de moins de 5000 habitants, à un Brutus mis en scène par Jean-Baptiste Reymond, " directeur de la comédie bourgeoise », auquel elle reconnaît du talent (il serait « assez fort pour jouer à Paris »). La jeune Riomoise, considérée comme affiliée, selon des termes empruntés au monde académique ou maçonnique, est installée à la place d'honneur dans la petite salle où l'on joue. Elle n'évite pas, dans son jugement final qui égratigne les comédiennes, leur accent, leur vulgarité, comme dans son refus de s'associer à elles pour une prochaine

39 R. Bouscayrol, Les lettres de Miette Tailhand-Romme : 1787-1797, [sans éditeur], Aubière 1979 ; Lettre 97, du troisième trimestre 1794 (« Le public a accueilli favorablement des amateurs qui voulaient bien prendre la peine de l'amuser. Il a applaudi à outrance ce qu'il aurait peut-être siffé dans une autre circonstance »), et Lettre 98, de novembre 1794. 
représentation ( « un honneur qui aurait compromis la dignité de notre société et peutêtre [sa] réputation $»^{40}$ ), une condescendance certaine. La demoiselle est moins sûre de son art que de la supériorité intellectuelle et professionnelle de ses parents et amis, consciente aussi d'une gradation dans les exercices de civilité et de la valeur des amateurs riomois. Dans ce cas précis et dans cette forme de sociabilité, la Révolution n'efface pas la hiérarchie des fortunes et des convenances.

Si nombre de ces cénacles amateurs, par leur sociologie comme par leur répertoire, doublent un temps le réseau jacobin, ils sont récupérés à partir de l'an III, du moins pour ceux qui perdurent, par la réaction thermidorienne, voire les royalistes. Les épurations administratives et politiques entraînent en effet une instabilité des troupes d'amateurs, et en transforment çà et là en l'an III la raison sociale et politique. Les interventions de la force armée se multiplient de l'an III à l'an $\mathrm{V}$, en proportion de la reconquête des théâtres par la réaction royaliste - à Dijon, Tarbes, Troyes, etc. Certains sont autoritairement fermés après le coup d'État républicain du 18 fructidor an $\mathrm{V}^{41}$. Ce temps des amateurs engagés a, par ailleurs, éclipsé nombre de troupes professionnelles, en perturbant les circuits, les obligeant à des amalgames temporaires avec leurs imitateurs, ou contraignant les artistes à exercer pour survivre plusieurs métiers en parallèle (à Dijon, à Nantes). Au-delà des productions locales ou adaptées aux circonstances, plébiscitées pour les unes par des amis bienveillants, pour les autres par des auditeurs loyalistes, les succès des amateurs sont souvent fondés sur les reprises de ceux du théâtre professionnel. Ouvrant la boîte des souvenirs privés, George Sand se souviendra avec précision de Robert, chef des brigands, imité de Schiller par Lamartelière, dans l'Histoire de ma vie ( $1^{\text {ère }}$ partie, chapitre VII). En l'an VI, son père, qui avait vingt ans et serait bientôt touché par la conscription, jouait le rôle de Robert en sa demeure de La Châtre, et mettait en scène ses partenaires. La société des amateurs de La Châtre, forte d'un orchestre, se donnait à voir en la circonstance dans la salle en gradins de l'ancien club jacobin, ci-devant couvent des Carmes, et s'était associé le secours des prisonniers de guerre hongrois et croates en cantonnement dans le district (pied de nez à la vie : le père de Sand sera quelques années plus tard prisonnier des Croates ...) : « On leur fit comprendre qu'après la bataille ils devaient paraître blessés ; ils se concertèrent si bien et y mirent tant de conscience qu'à la représentation on les vit sortir de la mêlée boîtant tous du même pied ».

\section{LES PUBLICS}

L'invitation des foules dans des mises en scène revendiquant la véracité historique, ou dans des spectacles par et pour le peuple, notamment repérés à Paris

40 Ibidem, Lettre 83, de septembre 1793.

41 M.-E. Plagnol-Dieval, op. cit.; Ph. Bourdin, « Les théâtres de société sont-ils solubles dans la Révolution ?... », pp. 157-168; idem, «Drama societies in the French Revolution... », pp. 3-27. 
et à Rouen ${ }^{42}$, est lancée, non sans reconnaissance. Or la critique, majoritairement, regrette la démocratisation du public et le mélange des genres censé les abâtardir tous; elle y voit un avatar de la Révolution. Et sans doute est-elle suivie par une partie de l'opinion dont les hérauts rejettent la politisation du répertoire, quand ils n'émigrent pas. Les salles de spectacle s'affirment alors comme des lieux de sociabilité ouverts, sans la sélection ni les règles de distinction des salons - d'où l'incompréhension des habitués de ces derniers, qui fréquentent de plus en plus nombreux les scènes de la capitale après leurs goûters dans les deux dernières décennies de l'Ancien Régime. Elles demeurent à policer, et Jeffrey Ravel a déjà montré combien l'ordre des parterres est un enjeu séculaire pour les municipalités ${ }^{43}$. À partir de 1751, des soldats en faction toujours plus nombreux (184 pour la première du Barbier de Séville en 1776 !) sont placés dans les salles parisiennes, puis provinciales, ce qui du reste procure un nouveau motif de moqueries, d'insultes, d'altercations et de violences dans et en dehors de la salle, sans compter les luttes de prérogatives qui s'ensuivent entre municipalités et pouvoir central (à Angers, Beauvais, Bordeaux, Paris, etc.). Les spectateurs, d'origines diverses mais souvent unis dans le combat, ne comprennent pas que leurs distractions payantes puissent être ainsi surveillées et contraintes en un lieu où le jeu et l'émotion collectifs doivent prévaloir sur le cérémoniel, voire sur l'illusion, où l'on se donne à voir et à entendre autant qu'on observe et qu'on écoute. Le tout grossit les Anecdotes dramatiques, qui mesurent traditionnellement le dysfonctionnement, l'échec ou la transgression de la tripartition idéalisée de l'espace entre la scène, la salle et les coulisses, au gré des aléas du dialogue social entre les interprètes et leurs auditeurs ${ }^{44}$. Ces manquements spontanés et continus, compassionnels ou caustiques, vocaux ou gestuels, à un horizon utopique qui séparerait la fiction de l'espace réel, ces anomalies, ces détournements, nourrissent aussi le débat sur la nécessité de créer des places assises partout dans les théâtres et de repousser l'élément populaire au " poulailler », tel que l'expérimente l'architecte Ledoux à Besançon au mitan des années 1770 . Ce, au grand dam de Marmontel ou de Louis-Sébastien Mercier, qui jugent irremplaçables les émotions des foules bariolées, condensé d'une nation, partageant dans la promiscuité de la station debout toute la gamme des émotions devant le ridicule ou le pathétique. Et c'est bien au nom de cette nation portée par les premiers événements révolutionnaires, et dans le cadre de la lutte contre le féodalisme, que des fidèles de la Comédie-Italienne

42 I. Metha, « La chronique théâtrale comme instrument de l'éducation patriotique à Rouen (1792-1799)», [dans :] La Révolution française : la guerre et la frontière, M. Cubells (dir.), Éditions du CTHS, Paris 2000, pp. 355-362.

43 J.S. Ravel, The Contested Parterre. Public Theater and French Political Culture: 1680 1791, Cornell University Press, New York 1999.

44 S. Marchand, «L'espace de l'événement ou le théâtre des Anecdotes dramatiques », [dans :] La scène, la salle et la coulisse dans le théâtre du XVIII siècle en France, P. Frantz, Th. Wynn (dir.), Presses de l'Université Paris-Sorbonne, Paris 2011, pp. 279-291. 
obtiennent le 6 août 1789 que soient remisés les bancs du parterre installés deux ans auparavant ${ }^{45}$.

Dans Le Censeur dramatique, ou Journal des principaux théâtres de Paris et des départemens, qu'il fait paraître chaque décade à partir de 1797, Grimod de la Reynière, à l'aune de l'élitisme académique de ses confrères, regrette la théâtromanie déferlante qui a ouvert les salles de spectacle à « toutes les classes de la société », mues par « une fureur plus encore qu'un besoin », un « délire universel $»^{46}$. Le théâtre semble à ce descendant de riches fermiers généraux avoir remplacé les jeux du cirque, et il y retrouve cette passion supposée inhérente à un peuple dépourvu de raison, à la "populace » ainsi dénoncée par les tenants de l'ordre social et politique dès les premières journées révolutionnaires. De là, à ses yeux, un déclin du bon goût, une disparition des lumières, une démagogie des suffrages (qu'aucune connaissance du répertoire classique, aucune règle de grammaire, de versification, de genre ne conduit), susceptibles de pervertir les arts de la scène et l'esprit des dramaturges, et omniprésents dans les frasques des amateurs ou des boulevards. La soldatesque, la jeunesse, les ouvriers « de la dernière classe du peuple », des commis d'administration, " assemblage hétérogène et fétide », « cette introduction des bonnets rouges à la place des têtes pensantes », ne seraient capables que de " conversation obscène », de " langage grossier », dévoilant « corruption précoce » et rusticité ${ }^{47}$.

Aux délicatesses de l'Ambigu-Comique, Restif de la Bretonne, pourtant moins élitiste que le directeur du Censeur dramatique, oppose les facéties grossières et crapuleuses de Nicolet, les incitations aux vices et à l'alcool devant lesquelles s'esbaudissent avec indécence et scandale « des faquins, de la lie, des tailleurs, des cordonniers, des savetiers, des débardeurs », concluant : « la classe des marchands et même des artisans ne trouve rien là qui puisse la séduire $»^{48}$. Les farces de la foire Saint-Germain sont jugées « obscènes », « détestables » par Mercier « parce qu' on leur interdit tout ouvrage qui aurait un peu de sel, d'esprit et de raison ", au mépris du public qui devrait avoir droit au grand répertoire ; les programmations deviennent « ordurières", amorales et corruptrices sur les boulevards - sinon quelques pièces en un acte. «Les scènes changeantes et perpétuellement renouvelées », données tard dans la nuit, et les parades détournent de leur devoir des ouvriers attirés par les prix modiques, « la bouche béante », tandis que l'on met dans celle « des petites filles, encore dans l'enfance, des obscénités

45 J.S. Ravel, op. cit., p. 222.

${ }^{46}$ Le Censeur dramatique, ou Journal des principaux théâtres de Paris et des départemens 1 , 1797, p. 3.

47 Ibidem, p. 7.

48 R. de la Bretonne, Les Nuits de Paris, édition établie et présentée par D. Baruch, Robert Laffont, Paris 1990, p. 841. 
choquantes $»^{49}$. Le « mauvais goût », débusqué dans l'opéra-comique, le mélodrame, la parodie, la comédie-parade, est un leitmotiv de la correspondance de Jean-François de la Harpe avec le Grand-Duc de Russie et le comte Schowalow.

Mais cette critique professionnelle est surtout portée à la défense d'un académisme, d'un classicisme, qui la fondent et deviennent sa raison d'être, la mettant souvent en contradiction avec les attentes d'une opinion publique bien mieux formée qu'elle ne fait mine de le croire et très capable de construire un jugement sans les béquilles des censeurs. Le baron Grivel, spectateur assidu au Théâtre-Français des débuts de Talma et de $\mathrm{M}^{\text {lle }}$ Mars, des succès de Baptiste aîné, Chomel, Dugazon ou Dazincourt, exprime avec nostalgie dans ses Mémoires, évoquant le Paris de l'an III, l'heureux temps où il construisait son jugement et le mettait en débat :

Il n'y avait point alors de claqueurs en titre, et la division du travail n'avait point encore produit cette espèce jugée indispensable plus tard. Le public prononçait donc en toute liberté et d'après ses impressions du moment. On eût perdu son temps si on eût essayé de dominer par la claque, comme cela s'est pratiqué depuis. Les passions politiques entraient sans doute pour beaucoup dans les jugements, mais déjà commençait à poindre un jour nouveau, et une sorte de réaction littéraire se faisait pressentir. Il était clair que le bon goût frappait à la porte du temple, et qu'il ne tarderait pas à reprendre son empire, à faire justice des maximes révolutionnaires qui ne seraient pas énoncées en bon français. Aux coins des parterres se trouvait d'ordinaire une réunion d'amateurs, qui débattait le mérite des acteurs et des pièces et qui était religieusement écouté par les jeunes gens. Je puis dire que j'ai fait là une sorte de cours de littérature dramatique, qui me plaisait beaucoup ${ }^{50}$.

Issu du peuple et devenu maître-artisan, Jacques-Louis Ménétra, suffisamment introduit pour fréquenter les coulisses et ayant foulé lui-même la scène en amateur, emmène sa famille voir les Italiens ou le répertoire de l'Opéra-Comique, mais apprécie tout autant les danseurs de corde du boulevard du Temple ou les arlequinades de Chaumont ${ }^{51}$. La province n'est pas en reste. On sait Miette Tailhand, nièce du Conventionnel Gilbert Romme, sensible aux spectacles de la Comédie de Clermont-Ferrand, très fréquentée au demeurant et occasion d'un affichage social qui conduit l'art du paraitre ${ }^{52}$, voire des rixes sanglantes entre loges et parterre, auxquelles les futurs députés Gaultier de Biauzat et Couthon avaient pu assister en 1780 et qui se reproduiront en l'an $\mathrm{V}^{53}$. Le même Biauzat n'avait pas oublié l'année précédente d'inciter ses concitoyens auvergnats, ses amis de la municipalité de Clermont-Ferrand surtout, à imiter la mise en scène de L'Offrande à la liberté, créée par Gossec et Méhul à l'Opéra de Paris, le 30 sep-

49 L.-S. Mercier, Tableau de Paris, édition établie et présentée par M. Delon, Robert Laffont, Paris 1990, pp. 117-118 et 249-250.

50 Mémoires du vice-amiral baron Grivel, Plon, Paris 1914, pp. 27-29.

51 Journal de ma vie : Jacques-Louis Ménétra, compagnon vitrier au XVIII siècle, D. Roche (présentation), Montalba, Paris 1982, pp. 317-318.

52 R. Bouscayrol, Les lettres de Miette Tailhand-Romme..., Lettre 25, 28 juin 1788.

53 A. Bossuat, «Le théâtre à Clermont-Ferrand aux XVII ${ }^{\mathrm{e}}$ et XVIII ${ }^{\mathrm{e}}$ siècles », Revue d'histoire du théâtre, t. II, treizième année, 1961-1962 ; Ph. Bourdin, Des lieux, des mots, les révolutionnaires..., p. 54. 
tembre $1792^{54}$. Mais le divertissement a ses limites lorsque la réalité subsume toutes les fictions. Un simple volontaire aux frontières, fils de procureur et cousin de « Miette » Tailhand, rappelle ainsi à sa sœur, actrice en herbe, ce que raison veut dire, lui imposant de reconnaître l'existence d'un plus grand et plus important théâtre, celui des opérations :

Je ne m'étendrai pas davantage sur ce que nous pouvons souffrir. Le courage et l'espérance fait disparaître toutes nos souffrances. Tout en jouant ici mon rôle dans le tragique, naturel, je souhaite à tous nos parents et concitoyens la suite de leurs amusements civiques. J'espère qu'à mon retour, si je suis assez heureux pour le voir, je ne serai pas un acteur sans apprentissage. Nous n'avons point ici pour maître ni les Racine, ni les Corneille, ni les Voltaire ; mais, cependant, le théâtre ne laisse pas que d'être des plus fréquentés ${ }^{55}$.

Les militaires de la République, en garnison, en France comme à l'étranger, sont un public captif pour les salles de spectacle. Il faut bien tuer le temps, si possible intelligemment, pour nombre de fils de la bourgeoisie devenus officiers, se souvenant des enseignements familiaux et collégiens ; il faut apprivoiser les populations et avec elles partager; enfin, l'espoir existe de croiser l'autre sexe. En stationnement à Châlons-en-Champagne en 1792, le capitaine Gerbaud note ainsi, pour mieux conjurer son ennui : « Nous avons cependant comédie, nous sommes tous abonnés, mais encore n'y vient-il guère de dames ${ }^{56}$. Fils d'un avocat de Montluçon (Allier), Gilbert Favier prouvera son appétit tôt inculqué pour les arts de la scène partout où le porteront ses pas de volontaire dans les armées républicaines : il apprendra, pour mieux goûter les tréteaux européens, les langues des pays occupés, l'allemand et l'italien particulièrement, traduisant Les souffrances du jeune Werther ou assistant par exemple, en l'an VIII, à une représentation en langue vernaculaire de Misanthropie et repentir, de Kotzebue ${ }^{57}$. Les amours des tréteaux sont autant périlleuses que déçues. À peine sorti de son école militaire où le sentiment royaliste était répandu, le futur général Griois s'ennuie ferme fin août 1792 à l'Opéra, qui redonne pourtant le réputé Roland de Quinault, Marmontel et Piccini (créé en janvier 1778, il avait provoqué la colère des gluckistes), « soit qu'il fût réellement mauvais ou que la fatigue, la gêne » éprouvées « au parterre où l'on était alors debout » ne le lui fissent trouver tel ${ }^{58}$. Et que dire du célèbre général Lefebvre, accompagnant son supérieur Augereau, fort de son aide décisive au coup d'État du 18 fructidor an V, et hôte de la Comédie-Française dont il a exigé

54 Archives départementales du Puy-de-Dôme, F 156 (78), Lettre de Biauzat aux administrateurs clermontois, 25 nivôse an IV (15 janvier 1796).

55 R. Bouscayrol, "Deux lettres d'un volontaire riomois à sa mère ", Revue d'Auvergne 2, 1985, pp. 249-254, Lettre de Gilbert Chabory du 3 nivôse an III (23 décembre 1794).

56 Ibidem.

57 L. Duchet, Deux volontaires de 1791. Les frères Favier de Montluçon. Journal et lettres, [sans éditeur], Montluçon 1909. Héritier d'une sociabilité bourgeoise, Gilbert n'est pas moins sensible à Terpsichore et fondera à dessein une société de danse à Besançon, au sein de l'état-major où il est capitaine - le droit d'entrée en sera prohibitif.

58 Mémoires du général Griois, t. 1, Plon, Paris 1909, p. 22. 
deux pièces de Voltaire, Brutus et La Mort de César ? Après avoir refusé avec la violence du soudard de prendre dans sa voiture l'épouse d'un de ses subordonnés ( "Va te faire f[outre], nous ne sommes pas ici pour accompagner des femmes, et surtout la tienne, qui a les t[étons] mous !»), le voilà installé : " Lefebvre, qui n'avait aucune idée de la littérature, applaudissait de tout son cœur et de ses grosses mains, croyant que c'était une pièce de circonstance faite le matin ; il me donnait des coups de coude à chaque instant en me disant : " Dis donc, dis donc, quel est le b[ougre] qui a fait cela ? Est-il ici ? $»^{59}$.

Les archives policières ou municipales prouvent cependant que l'épisode révolutionnaire n'induit pas de vraie rupture dans les formes et la régularité des incidents qui émaillent nombre de représentations. Ces incivilités finissent même vraisemblablement par faire partie de l'horizon d'attente des spectateurs quand même elles ne sont pas organisées. Les théâtres marseillais nourrissent par exemple régulièrement l'anecdote. Treize affaires les agitent entre mars 1790 et novembre $1791^{60}$. Hormis les cas d'ébriété, les altercations nées de la promiscuité (un chapeau dont la pointe rentre dans l'œil du voisin), qui peuvent dégénérer en coups de bâton, les affaires strictement privées envahissent rarement l'espace. Il faut toute la malignité perverse de la très légère et vénale Julie Boucher pour mettre sous les yeux d'un vieil amant son substitut le plus jeune, leurs moqueries piquant au vif le cocu qui vient la souffleter, suscitant un murmure réprobateur dans la salle. Le collectif qui s'exprime en temps ordinaire prouve plutôt combien sont grandes les impatiences d'un public qui se fait censeur, prompt à réclamer un lever de rideau, l'engagement de ses artistes favoris (le comédien Porteuil, le maître de ballet Blache) ou la relégation des plus honnis (le médiocre Valville). Ses broncas affolent les officiers municipaux, les gardes, les policiers et leurs mouches disséminés dans la salle. Elles ne vont pourtant guère plus loin que des sifflets, des huées, des noms d'oiseaux, des mouchoirs agités, des jets d'objets sur la scène, des poses indécentes - excitant ses contemporains, la fille Barthe, par exemple, le postérieur nonchalamment appuyé sur l'accoudoir d'une loge, agite un grand mouchoir blanc : elle prétendra avoir trop chaud en position assise et faire un peu de ménage ! Les interpellations, les emprisonnements temporaires, les amendes (de 50 à 500 livres) les interdictions de paraître au spectacle (d'un à trois mois), et les sentences imprimées qui en résultent révèlent des intervenants socialement divers. Se bousculent " au violon » trois commis, deux marchands-négociants, des maîtres artisans (fabricant d'indiennes, balancier), un bourgeois d'Aix-enProvence, des manouvriers du port, deux prostituées, deux militaires (dont un Anglais), les artistes eux-mêmes - le malmené Valville ; le régisseur du Grand Théâtre Verteuil, qui a osé lire une lettre attachée à une couronne lancée sur la scène ; son successeur Quériot, dont le peu d'empressement à déférer aux désirs

59 Souvenirs du Maréchal Macdonald, Le Livre chez vous, Paris 2007, pp. 118-121.

${ }^{60}$ Archives municipales de Marseille, 1 I550. 
du public provoque des bagarres entre balcons et parterre ; la comédienne Victoire Duchêne, qui, abandonnant la scène dès les prémisses de ces troubles, encourage la panique et le baisser de la toile ${ }^{61}$. Si la gent militaire est souvent prise à partie, exceptionnels sont les troubles qui relèvent du politique. En mars 1791 cependant, le parterre du Grand Théâtre est agité par une altercation entre ceux qui crient : « Le Roi ! Le Roi ! », et ceux qui leur répondent : « Il n’y a plus de roi ! C'est la Nation! Paix ! À bas!». Un garde national descend des premières loges pour corriger un quidam du parterre qui a crié « À bas l'uniforme ! », et qui est défendu par « les bourgeois» voisins ${ }^{62}$.

Partout la remise dans le droit chemin de la civilité se fait par affiches dénonciatrices, par la proclamation répétée des règlements ou des amendements à ces derniers, dont la lecture en creux nous en dit long sur les agitations quotidiennes. L'exposé prolixe produit en brumaire an V par la municipalité de Castres est, de ce point de vue, révélateur. Il mêle édification privée et morale publique : « la protection qui est düe à des établissements où l'attrait du plaisir appelle nécessairement un concours considérable de citoyens de tout sexe et de tout âge, exige encore, pour avoir un but vraiment utile, que les magistrats veillent à ce qu'il ne s'y pratique rien de contraire aux mœurs, à l'esprit public, à l'union des citoyens ». Il interdit, en conséquence, « les clameurs, les dénominations injurieuses, les allusions perfides, les chants de haine qui rappellent ou le règne des factions ou de douloureux souvenirs, et qui entraînent le plus souvent des chocs d'opinion et des rixes qui dégénèrent avec trop de facilité en affaires de parti ». Représentations et répertoires (y compris celui des hymnes entonnés à l'entracte) seront soumis à une autorisation municipale, toute adaptation et détournements ultérieurs d'un texte admis lourdement sanctionnés, toute adresse aux artistes interdite (interpellation, murmures ou cris de toutes espèces). Une seule attitude : « la décence et la tranquillité que doivent scrupuleusement observer les amis des mœurs et du bon ordre ». De là, la déclinaison des nombreuses entorses à la règle :

11. [...] il est fait déffences à ceux qui assisteront au spectacle d'avoir le chapeau sur la tête tant que la toile sera levée, de rien placer sur les accoudoirs des loges, de tourner le dos au théâtre, de se tenir debout au parquet et sur le devant des loges, de garder aucune place en y tenant le chapeau, de fumer, de battre la mesure du pied, de faire du bruit dans le passage des loges, ou en parlant de manière à priver leurs voisins du plaisir d'entendre les acteurs. Ceux qui n'écouteroient point la première sommation qui leur serait faite pour les rappeler à l'ordre et au silence, seront expulsés de la salle et même rendus responsables et traités comme provocateurs du trouble qui pourrait avoir lieu.

12. Il est également déffendu à ceux qui se placent au parterre de pousser et repousser leurs voisins dans aucun sens, ni de faire aucun mouvement forcé pour exciter un flux et reflux, ou pour produire par ces pressions aucun vuide dans le parterre, à peine pour les contrevenans d'être arrêtés et poursuivis comme perturbateurs.

13. Les mêmes mesures seront prises contre ceux qui jetteroient sur le théâtre ou dans la salle des

${ }^{61}$ Bibliothèque municipale de Marseille, 1I550, Délibération municipale du 17 octobre 1791.

${ }^{62}$ Bibliothèque municipale de Marseille, 1I550, Délibération municipale et affiche du 17 mars 1791. 
oranges, des pommes ou d'autres objets, et contre ceux qui, par un badinage hors de saison, troubleroient quelque spectateur.

14. Deffenses sont pareillement faittes à toutes personnes d'introduire dans la salle du spectacle aucun chien ou autres animaux sous les peines de droit.

15. Il est encore déffendu à tous citoyens, autres que les artistes, et ceux qui doivent concourir avec eux à l'exécution des pièces, d'entrer dans le foyer, de se placer pendant le spectacle et les entractes sur le théâtre et dans les coulisses, ce qui nuit à l'illusion, distrait les acteurs, les empêche de se préparer et excite le plus souvent des murmures de la part des spectateurs. À cet effet, il est deffendu aux servans du théâtre de fournir ou laisser placer aucune chaise dans les coulisses.

16. Toute personne qui porterait ou se servirait au spectacle de chaufferettes sera poursuivie pour son imprudence d'après la rigueur des loix de police.

17. L'entrée des salles de spectacle, bals, concerts et autres lieux pareils, est interdite aux enfants qui ne seroient pas conduits par leurs parens ou des personnes capables de les représenter, et à tout individu déguisé ou masqué, indécemment habillé ou en état d'ivresse, ou qui ne serait pas décoré de la cocarde nationale. L'entrée desdits lieux est en outre interdite à ceux qui refuseroient de payer ou de se soumettre au règlement fait pour y être admis ${ }^{63}$.

Particulièrement nombreuses en l'an V et l'an VI, ces motions d'ordre sont également révélatrices des luttes politiques fratricides dont les salles ont été les écrins, soit à l'heure de la reconquête royaliste par les urnes, soit à l'heure de la réaction républicaine, après le coup d'État du 18 Fructidor ${ }^{64}$. Les mêmes précautions prévalent donc, précisées, à Albi, en trois textes successifs de l'an IV à l'an VI, et en quelques autres mesures contre les troupes itinérantes, suspectées d'abriter réquisitionnaires et émigrés ${ }^{65}$. Le répertoire des chants républicains autorisés, et entonnés sur l'avant-scène avec accompagnement orchestral, y est précisé ( $L a$ Marseillaise, Ça ira, Veillons au salut de l'Empire, Le Chant du Départ), avec obligation pour le public de l'avoir appris auparavant pour éviter tout désordre - il sera donc indiqué sur les affiches. Toute chanson intempestive, tout sifflet, toute huée, toute insulte qui interromprait le jeu des artistes vaudra emprisonnement immédiat ; tout désordre sera imputé aux propriétaires de la salle, qui sont invités à laisser le moins de temps mort possible entre les deux pièces d'une même soirée. L'univers des coulisses est désormais inaccessible : nul spectateur ne peut franchir cet espace, nul accessoiriste se montrer sur scène ${ }^{66}$. La tenue même du public albigeois est vérifiée, tant l'habit et la mise se confondent, en Révolution, avec l'affichage des opinions. Ce qui n'évite rien... À la fin de messidor an VI,

${ }^{63}$ Archives municipales de Castres, 1D 5, Délibération municipale du 15 brumaire an V (5 novembre 1796).

${ }^{64}$ Cf. C. Tréhorel, Le théâtre de Dijon de 1789 à 1810, [mémoire de maitrise de l'Université de Bourgogne], 1999 ; J. Horn, " La lutte des factions au théâtre de Troyes sous le Directoire », [dans :] La République directoriale, t. 2, Ph. Bourdin et B. Gainot (dir.), Presses universitaires Blaise-Pascal, Clermont-Ferrand 1998, pp. 679-690.

65 Archives départamentales du Tarn, L 210, $\mathrm{f}^{\circ}$ 18, Lettre du ministre de la Police générale au commissaire du pouvoir exécutif près l'administration centrale du département, 11 pluviôse an IV (31 janvier 1796).

66 Archives municipales d'Albi, 1D 4, Délibérations municipales du 27 germinal an IV (16 avril 1796), du 5 vendémiaire an V (28 septembre 1796), du 25 messidor an VI (13 juillet 1798). 
plusieurs altercations perturbent la salle de spectacle, motivées par la présence de jeunes Muscadins, " avec leurs cheveux coupés et hérissés à la Caracalla ${ }^{67}$.

Le théâtre fait donc société, ce que dirait d'une autre façon l'étude du répertoire. Il fait société sur les plans économiques, sociaux, et politiques, mais aussi dans les combats pour les normes - esthétiques, civiques et civiles — dont il est l'écrin et le creuset. La loi du 13 janvier 1791, les préconisations ultérieures des pouvoirs publics lui conférant une haute vertu éducative, le marquent indéniablement, non seulement pour leurs conséquences sur les salles, le répertoire et l'organisation des professionnels, mais aussi parce qu'elles sortent les théâtres de société de leurs étroits cercles sociaux originels pour les projeter vers un public plus large, vers une mission citoyenne, ou qu'elles associent les spectateurs aux expériences par et pour le peuple. La Révolution n'efface pourtant pas la hiérarchie des fortunes et des convenances, ni les processus de discrimination à l'œuvre sous l'Ancien Régime, dont Grimod de la Reynière est un pur produit. Le sûr jugement des critiques, le " gros goût bourgeois ", l'indécence populaire supposée forment trois entrées d'un discours sur la distinction, à géométrie variable de la province à Paris, au travers duquel se maintiennent ou s'affirment des dominations sociales. Pour reprendre Pierre Bourdieu : « La négation de la jouissance inférieure, grossière, vulgaire, vénale, servile, en un mot naturelle, qui constitue comme tel le sacré culturel, enferme l'affirmation de la supériorité de ceux qui savent se satisfaire des plaisirs sublimés, raffinés, désintéressés, gratuits, distingués, à jamais interdits aux simples profanes $»^{68}$. Loin du sensationnel ou de l'émotionnel appréciés d'une grande partie du public, le souci éducatif, le souci d'ordre moral et politique, surtout à partir de l'an III et du déclin de la sans-culotterie, contribuent à une séparation revendiquée entre la salle et la scène, qu'entérinent les règlements municipaux : le spectacle devient une grand-messe à laquelle le public devrait communier en silence. Les « incivilités » sont d'autant plus vivement dénoncées, les « obscénités » idéalement renvoyées au poulailler. Ce processus de sacralisation du texte, des artistes, la partition des espaces et la pacification des parterres prendront cependant plus d'un siècle pour aboutir, au prix de nombreuses altercations collectives et spontanées ${ }^{69}$.

67 Archives départamentales du Tarn, 1207, $\mathrm{f}^{\circ}$ 133-134, Lettre du 26 messidor an VI (14 juillet 1798) du commissaire du pouvoir central auprès du département au ministre de la Police générale.

68 P. Bourdieu, La Distinction. Critique sociale du jugement, Les Éditions de Minuit, Paris 1979, p. VIII.

69 J.-C. Yon, « Du droit de siffler au spectacle au XIX ${ }^{\mathrm{e}}$ siècle », [dans :] La voix et le geste. Une approche culturelle de la violence socio-politique, Ph. Bourdin, J.-C. Caron et M. Bernard (dir.), Presses universitaires Blaise-Pascal, Clermont-Ferrand 2005, pp. 321-337. 


\section{THEATRE AND SOCIETIES IN REVOLUTIONARY FRANCE}

\section{Summary}

The theatre, a very often-frequented place from the 1770 s, is at the junction of several societies: that of the shareholders who own the auditorium and privileges; that of the artists; that of the spectators; that of amateurs who are formed in bourgeois circles and then in patriotic dramatic societies. Commercial freedom, activist investment, the wars born of the French Revolution, emigration, indeed, upset theatrical structures. Halls and troops then multiply, and dramatic practices gain previously unfamiliar spaces for entertainment. Theatre becomes not only an economic issue, but also a political one, posing and addressing long term issues of profitability, social order, and public order. Theatre enables social reconversions, but professional troops are also sometimes divided by the artists' political choices. They are challenged by amateurs whose commitments are more in line with the wishes of the successive regimes.

Key words: theatre, French Revolution, public order, social order, censorship, shareholders, civility, sociability, Police of entertainment. 